\title{
Harninkontinenz individuell diagnostizieren und interdisziplinär behandeln
}

- Harninkontinenz gehört zu den Volkskrankheiten mit steigender Prävalenz im Alter, berichtete Prof. Hubertus Riedmüller, Würzburg. Mit $20 \%$ versus $10 \%$ klagen mehr Frauen als Männer jenseits von 60 Jahren über eine Harninkontinenz - laut Riedmüller ein Ausdruck dafür, dass die Tabuisierung der Problematik in der männlichen Bevölkerung deutlich stärker ausgeprägt ist. Bisher werden nur $10 \%$ der Hilfesuchenden behandelt, kritisierte Riedmüller.

Angesichts der Komplexität der Harninkontinenz plädierte er für ein individuelles diagnostisches Vorgehen in den verschiedenen Altersgruppen mit einer umfassend und sorgfältig durchgeführten Blasenfunktionsprüfung als wesentlichem Bestandteil. Die unterschiedlichen Kontinenzformen sind am ehesten Erfolg versprechend zu behandeln, wenn - je nach Einzelfall - Gynäkologen, Urologen, Chirurgen, Neurologen, Physiotherapeuten, Ernährungsmediziner, Sexualmediziner und Experten aus der rehalbilitativen Medizin nach neuesten wissenschaftlichen Erkenntnissen zusammenarbeiten.

Bei überaktiver Blase sollte zum Beispiel grundsätzlich mit konservativen Methoden wie Beckenbodentraining begonnen werden. Erst bei Versagen dieser Verfahren seien blasendämpfende Medikamente oder eine Injektion von Botulinumtoxin in die Blasenwand indiziert.

Ute Ayazpoor

Pressekonferenz im Rahmen des 24. Jahreskongresses der Deutschen Kontinenz Gesellschaft 2012, Würzburg, 9.November 2012, Veranstalter: Deutsche Kontinenz Gesellschaft
Änderung bei

\section{Finasterid-Filmtabletten}

Die ursprünglich blauen Filmtabletten Finasterid Winthrop ${ }^{\circledR} 5$ mg mit der Prägung „F5“ und einem Durchmesser von $7 \mathrm{~mm}$, die zur Behandlung der benignen Prostatahyperplasie eingesetzt werden, sind nun gelb und haben einen Durchmesser von 7,1 $\mathrm{mm}$. Das blaue Indigocarmin wurde dabei durch gelbes Eisenhydroxidoxid ersetzt und auch andere nicht wirksame Bestandteile der Filmtabletten haben sich geringfügig geändert. Die galenischen Eigenschaften der Filmtabletten sowie Wirkstoffgehalt, Wirkstoffstabilität und Wirkstofffreisetzung bleiben durch diese Änderungen unbeeinflusst, ebenso bleiben die Packungen und Pharmazentralnummern unverändert.

Während des Abverkaufs der restlichen Chargen mit alter Farbe und Größe werden die beiden Formen parallel im Handel sein.

Nach Informationen von

Winthrop, Mülheim-Kärlich

\section{Exakte Biopsie für sichere Diagnose}

Die Qualität der Tumordiagnostik kann für die Wahl der individuell bestmöglichen Therapie des Patienten entscheidend sein. Das neue CORAZOR-Schussgerät, das auf die CORAZOR-Stanzbiopsiekanüle abgestimmt ist, optimiert das Diagnoseverfahren und liefert exakte Ergebnisse für die histologische Untersuchung auf Tumoren der Prostata.

Aufgrund der speziellen Geräteform ist sowohl das Spannen als auch das Auslösen ohne Umgreifen möglich, eine Führungsschiene sorgt für eine besonders stabile Lage der Kanüle im Gerät. Eine Deckelsicherung verhindert das versehentliche Öffnen. Als einziges MehrfachSchussgerät auf dem Markt ist das CORAZOR-Schussgerät halbautomatisch. Dies ermöglicht es, zunächst die Innenkanüle und im zweiten Schritt die Außenkanüle vorzuschießen, die das Biopsat schneidet. Dadurch drückt sich das kurzzeitig verdrängte Gewebe in die freiliegende Kerbe und die Ausbeute des zu untersuchenden Materials kann verbessert werden.

Nach Informationen von UROMED, Oststeinbek tember 2012 eine neue Folgetherapie zugelassen, die in den ESMO-Leitlinien ebenfalls den höchsten Evidenzgrad erhielt. Axitinib ist tät. Axitinib verlängerte das mediane progressionsfreie Überleben sowohl nach einer Vorbehandlung mit Sunitinib (4,8 vs. 3,4 Monate) als auch nach einer vorherigen Zytokintherapie (12,0 vs. 6,6 Monate) im Vergleich zu Sorafenib. Eine signifikante Verbesserung um mehr als zwei Monate (6,8 vs. 4,7 Monate) zeigte sich auch in der Gesamtpopulation.

Nach Informationen von Pfizer, Berlin 